\title{
Current status of preoperative biliary drainage
}

\author{
Junko Umeda ${ }^{1}$ Takao Itoi $^{1}$
}

Received: 7 June 2015/Accepted: 10 June 2015/Published online: 3 July 2015

(C) Springer Japan 2015

\begin{abstract}
Preoperative biliary drainage (PBD) has been thought to be preferable regardless of the site of biliary strictures, e.g., distal or proximal strictures because PBD by endoscopy or interventional radiology decreases postoperative mortality and morbidity rates. However, recently, several studies have revealed that PBD strategy showed an increased mortality rate or a high frequency of surgical site infection. Herein, we reviewed reports in the literature regarding the current status of $\mathrm{PBD}$ and investigated the effects of PBD on patients with distal and proximal biliary obstructions due to potentially resectable pancreatobiliary cancers. Our summary demonstrated that there is as yet no optimal PBD method regardless of the distal and proximal biliary strictures because of the small sample size and the lack of better control groups in previous studies. Thus, prospective randomized studies with a large sample size are needed to establish the optimal mode of PBD and to evaluate the potential benefits of PBD in patients with both distal and proximal biliary obstructions.
\end{abstract}

Keywords Preoperative biliary drainage - Distal biliary strictures $\cdot$ Proximal biliary strictures

Part of this review was presented at The 4th International Forum of the 100th General Meeting of the Japanese Society of Gastroenterology.

Takao Itoi

itoi@ tokyo-med.ac.jp

1 Department of Gastroenterology and Hepatology, Tokyo Medical University, 6-7-1 Nishishinjuku, Shinjuku, Tokyo 160-0023, Japan

\section{Introduction}

Preoperative biliary drainage (PBD) has been developed since the 1960s to improve the postoperative morbidity and mortality rates of jaundiced patients with pancreatobiliary cancers. Obstructive jaundice and the following hyperbilirubinemia were identified as risk factors for perioperative complications because of physiological disturbances such as impaired immune response, coagulopathy, and liver, kidney, and other organ dysfunction besides severe pruritus.

To date, several experimental studies and retrospective case series have revealed that percutaneous or endoscopic PBD decreases postoperative mortality and morbidity rates [1-5]. In some studies of PBD strategy, an increased mortality rate or a high frequency of surgical site infection has been observed [6-9]. Although PBD has been routinely conducted to ensure safe anesthesia and operation, the beneficial effects of PBD remain uncertain. In addition, even if PBD is performed, the selection of either a plastic stent (PS) or a metal stent remains controversial. Furthermore, the effectiveness of PBD may depend on the type of cancers (e.g., pancreatic cancer and cholangiocarcinoma) or the site of biliary strictures (e.g., distal or proximal strictures).

In this article, we reviewed reports in the literature regarding the current status of PBD and investigated the effects of PBD on patients with distal and proximal biliary obstructions due to potentially resectable pancreatobiliary cancers.

\section{Preoperative biliary decompression}

At present, there are two main biliary drainage techniques, namely, (1) endoscopic drainage including nasobiliary drainage and biliary stenting (plastic or metal) and (2) 
percutaneous transhepatic drainage (internal or external). Recently, owing to the improvement of endoscopic procedures, endoscopic biliary drainage has been commonly used across the world. Jinkins et al. [10] evaluated the current trends in PBD at the population level and demonstrated that the use of preoperative biliary stenting doubled between 1992 and 2007 despite evidence of increasing perioperative stenting-related infectious complications. The increase in preoperative biliary stenting was due to the increased use of endostenting with endoscopic retrograde cholangiopancreatography (ERCP); percutaneous stenting was used at a fixed rate. Moreover, evaluation by a gastroenterologist before a surgeon was associated with an increased likelihood of endostenting. Thus far, selection of the PBD method has depended on the preference and experience of endoscopists or gastroenterologists. In addition, the latest development of stent devices (e.g., removable metal stents, antireflux stents, and absorbable stents) complicates the establishment of a standard PBD for pancreatobiliary cancers.

The preference for either percutaneous drainage or endoscopic drainage remains unclear, although a prospective randomized study favored the endoscopic approach [11]. Considering the patient's quality of life, endoscopic stent placement appears to be less invasive than percutaneous transhepatic drainage, although endoscopic biliary stenting (EBS) may cause complications such as pancreatitis, cholangitis, bleeding, and perforation. A prospective study revealed that PBD with endostenting results in almost $100 \%$ bacterial contamination of the bile ducts, and postoperative infectious complications have been associated with bile bacteribilia after PBD [12]. This has been regarded as a main factor affecting perioperative outcome with endostenting. Percutaneous transhepatic drainage also carries a risk of bleeding, bile leakage, and liver abscess. Among other things, the most worrying complication is tumor seeding along the catheter tract. The main advantage of an endoscopic approach over percutaneous intervention is the avoidance of skin and liver punctures in patients with deep jaundice and underlying coagulopathy, and tumor seeding.

\section{PBD for distal biliary obstruction (Figs. 1a, b, 2a, b)}

The majority of patients with periampullary cancer, including the head and neck of the pancreas, the distal end of the bile duct, and the ampulla of Vater, suffer from symptomatic obstructive jaundice due to distal biliary obstruction. For these patients, surgical resection is the only curative option, and approximately 15 to $20 \%$ of the patients are candidates for curative-intent surgery by pancreatoduodenectomy [13-16]. The perioperative morbidity rate still remains high despite a significant improvement in the postoperative mortality rate over the last decades, from more than $20 \%$ down to $2.9 \%$ [17]. To date, PBD has been performed to correct alterations and functional impairment induced by jaundice, to prevent cholangitis after diagnostic ERCP, to improve hyperbilirubinemia because of an expected delay in surgery, or allow the delivery of neoadjuvant therapy. As described previously, some experimental animal studies have shown the benefits of PBD, particularly after internal drainage when the enterohepatic circulation was restored $[18,19]$. The retrospective studies and a randomized control study published in the 1980s have also shown decreased mortality and morbidity rates in patients who underwent drainage [5, 20-24]. However, clinical studies have failed to show this benefit, and some studies even reported a deleterious effect (Tables 1, 2, 3). Povoski et al. [6] conducted a retrospective review of 240 cases of PD (PBD $73 \%$ ) with intraoperative bile culture data, and showed that PBD was associated with increased rates of positive intraoperative bile culture and infectious-related complications, leading to a four-times greater mortality rate. In another retrospective review of 567 patients who had undergone PD (PBD $72 \%$; PTBD $64 \%$, EBS $36 \%$ ), Sohn et al. [7] found that PBD was associated with an increased rate of wound infection and pancreatic fistula; however, the overall complication and mortality rates did not differ according to the procedure. In 2001, Pisters et al. [8] reported a retrospective review of 300 cases of PD in which $57 \%$ received PBD, $12 \%$ underwent surgical bypass, and $31 \%$ received no PBD. Moreover, PBD was associated with an increased risk of wound infection; however, the overall complication rate did not differ between groups. These results may justify the use of PBD to create time for the referral of patients to high-volume centers.

On the other hand, Strasberg et al. [25] evaluated 30 possible survival risk factors in a database of more than 400 resected patients. They reported that preoperative jaundice is a negative risk factor in patients with adenocarcinoma of the pancreatic head. Jaundiced patients who underwent preoperative stenting showed a survival advantage.

The latest Cochrane database systematic review [26] on this topic included six randomized control studies consisting of four trials using percutaneous transhepatic biliary drainage (PTBD) and two trials using endoscopic sphincterotomy and stenting (ES) as the PBD method. From the aspect of mortality, there was no significant difference in the overall mortality rate between the PBD group and the direct surgery (DS) group (RR 1.12; $95 \%$ CI 0.73-1.71; $p=0.60$ ). The overall serious morbidity rate was higher in the PBD group (RaR 1.66; $95 \%$ CI 1.28-2.16; 
a

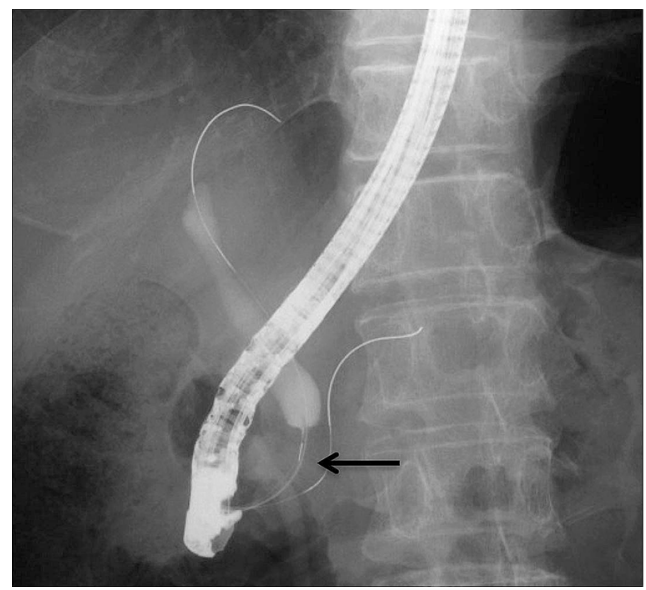

b

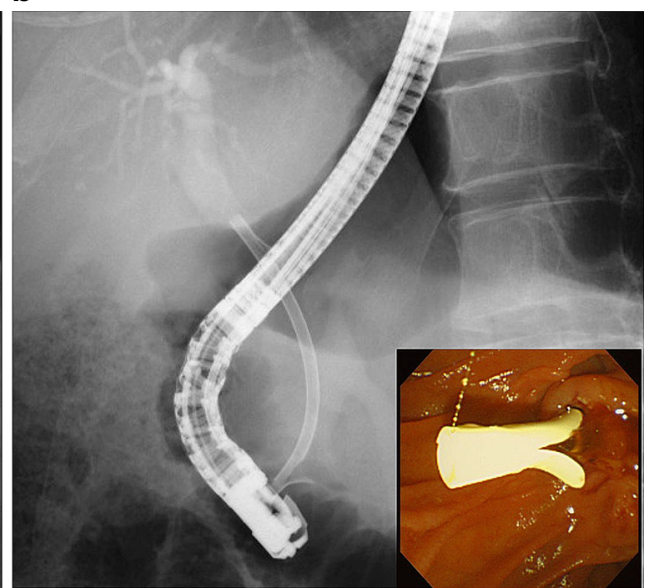

Fig. 1 Cholangiogram shows distal biliary stricture due to pancreatic cancer (arrow) (a). A fluoroscopic imaging (b) of the plastic stent placed across the distal biliary stricture (right lower corner; endoscopic imaging)

a

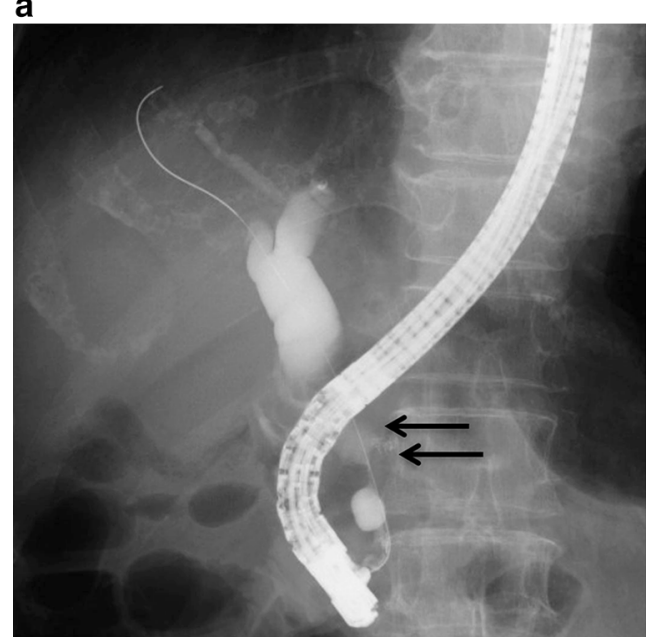

b

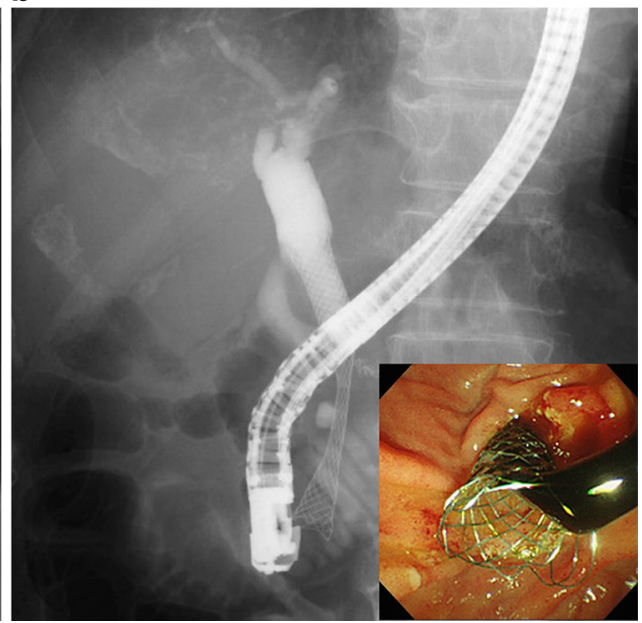

Fig. 2 Cholangiogram shows distal biliary stricture due to pancreatic cancer (arrow) (a). A fluoroscopic imaging (b) of the metal stent placed across the distal biliary stricture (right lower corner; endoscopic imaging)

$p=0.0002)$ than in the DS group. Although the quality of the trials was relatively high, the results cannot be estimated as representative of the current status of PBD because of the heterogeneity of the drainage techniques, stent selection, obstruction sites, and operative procedures.

A recent meta-analysis by Garcea et al. [27] consisted of 30 retrospective studies and six randomized control studies published between 1981 and 2008. The meta-analysis detailed the outcomes of 5133 patients, focusing on the effects of PBD on mortality, morbidity, and bile cultures. The authors found that PBD significantly increased the rates of bile culture positive for bacteria and the probability of wound infection $(p<0.0005)$. However, in keeping with previous studies, no evidence has been found to show that PBD directly increases postoperative mortality and morbidity rates. It is possible that in certain patients, PBD may deleteriously affect the outcome by bacterial contamination of the bile.

Velanovich et al. [28] examined previous studies published from 1994 to 2006, and reported on patients receiving only $\mathrm{PBD}$ with stenting for a distal biliary obstruction and who underwent pancreatoduodenectomy. The authors also conducted a cohort analysis of 181 patients who underwent pancreatoduodenectomy at their institution, and concluded that PBD increased the postoperative wound infection rate by about $5 \%$. However, there is no overwhelming evidence that PBD either promotes or protects patients from other complications.

Based on the results of currently available meta-analyses, the routine use of PBD has no beneficial effects on patients with periampullary cancer. However, the outcome measures have not yet been standardized and the lack of 


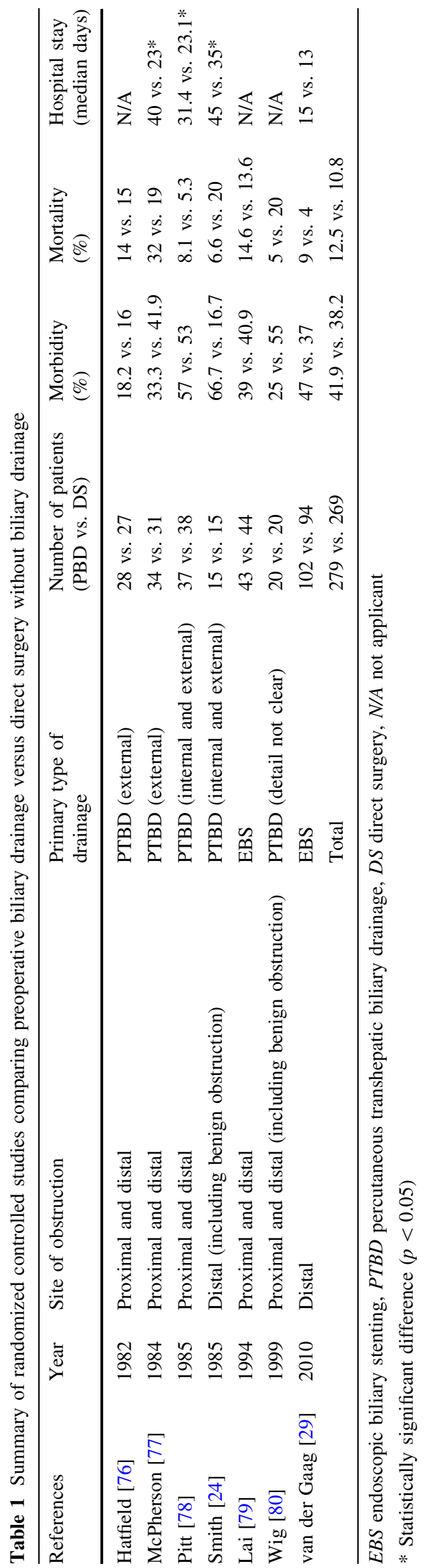

complete data on complications following PBD make direct comparisons difficult and biased.

In 2010, van der Gaag et al. [29] reported the results of a randomized multicenter study comparing PBD followed by surgery with surgery alone for patients with pancreatic head cancer. Patients enrolled with a serum total bilirubin level range of 2.3 to $14.6 \mathrm{mg} / \mathrm{dl}$ were randomly assigned either to undergo PBD for 4-6 weeks or to proceed to surgery within 1 week. PBD was attempted primarily with the placement of a 7-Fr PS via ERCP and, if not successful, by a second endoscopic attempt or a percutaneous approach at a tertiary referral center. The study endpoints were effective amelioration of the biliary obstruction and reduction of severe complications within 120 days after randomization. Mortality and the length of hospital stay were also evaluated. The study enrolled 202 patients with 6 patients excluded from analysis; 94 patients were assigned to undergo early surgery and 102 patients to undergo PBD. PBD was successful in $94 \%$ of the patients after one or more attempts, with a drainage-related complication rate of $46 \%$. The mortality rate and the length of hospital stay were not significantly different between the two groups. The surgery-related complication rate was $37 \%$ in the early surgery group versus $47 \%$ in the PBD group (relative risk, $0.79 ; 95 \%$ CI $0.57-1.11 ; p=0.14$ ). The rates of the overall complications including both drainage-related and surgery-related complications were significantly higher in the biliary drainage group than in the surgery group (39 vs. $74 \%$ relative risk in early surgery, $0.54 ; 95 \%$ CI $0.41-0.71 ; p<0.001)$. They concluded that PBD using a PS was not recommendable owing to procedure-related complications such as pancreatitis, bleeding, perforation, and cholangitis. This data supported the prior studies suggesting that PBD should not be performed routinely. However, there were major concerns in this study. Firstly, the failure rate for the initial ERCP and the rate of procedure-related complications appeared to be higher than the rates reported previously for high-volume centers (5-10\% for both) [30]. Secondly, patients with severe bilirubinemia (serum bilirubin $>14.6 \mathrm{mg} / \mathrm{dl}$ ) who may have derived the greatest PBD benefit were excluded. These patients were at substantially higher risk for cholangitis, liver dysfunction, and coagulopathy. Finally, the use of a 7-Fr PS was closely associated with early stent occlusion during the waiting period for operations. This might increase the rate of drainagerelated complications as larger PSs or self-expandable metal stents (SEMSs) have a longer patency period; 2-5 months for $10-$ Fr PSs [31, 32] and 5-12 months for covered or uncovered metal stents [33]. Baron and Kozarek suggested designing similar studies for evaluating the placement of short $(4-6 \mathrm{~cm})$, uncovered, or fully covered SEMSs [34]. 


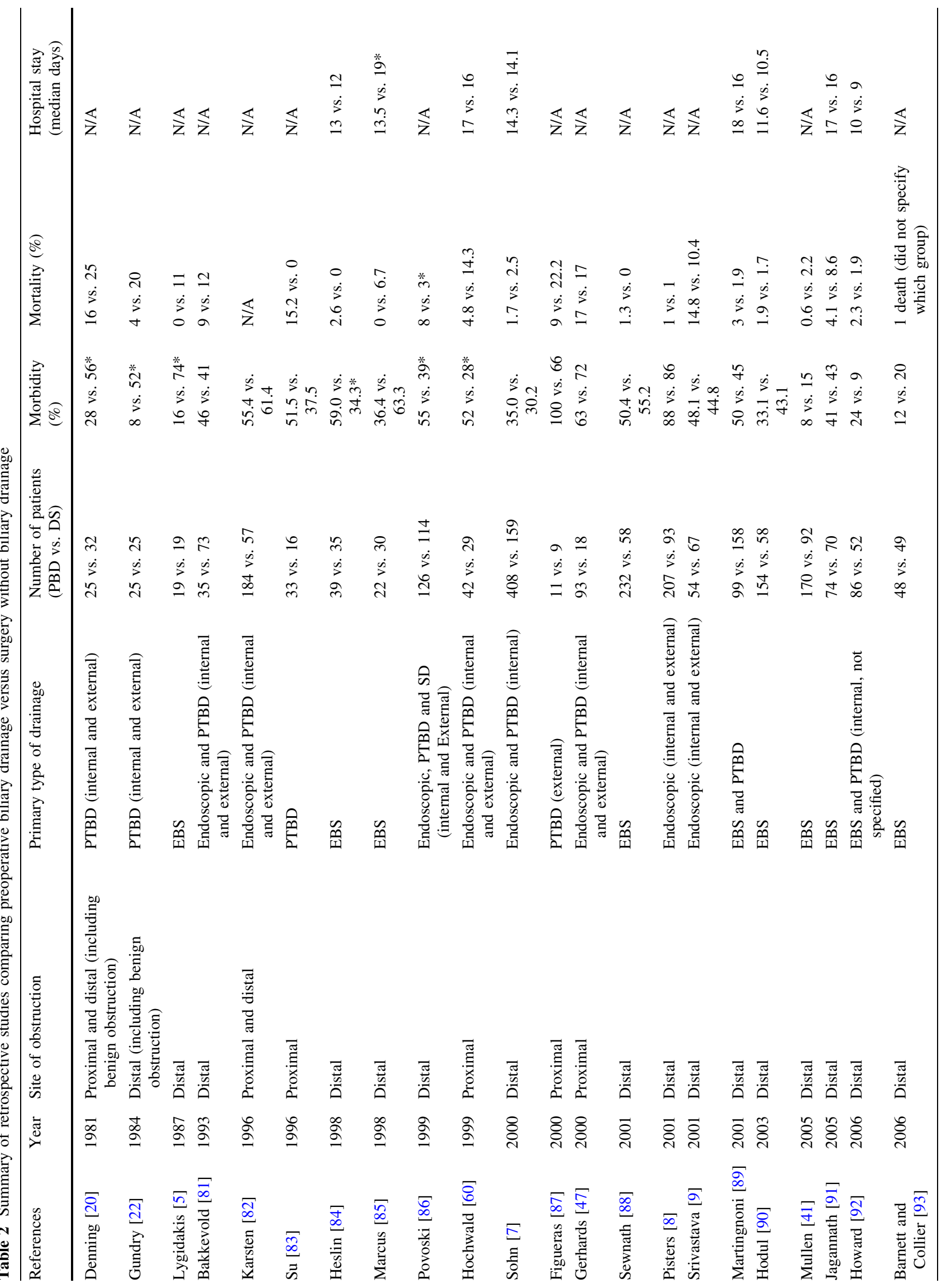




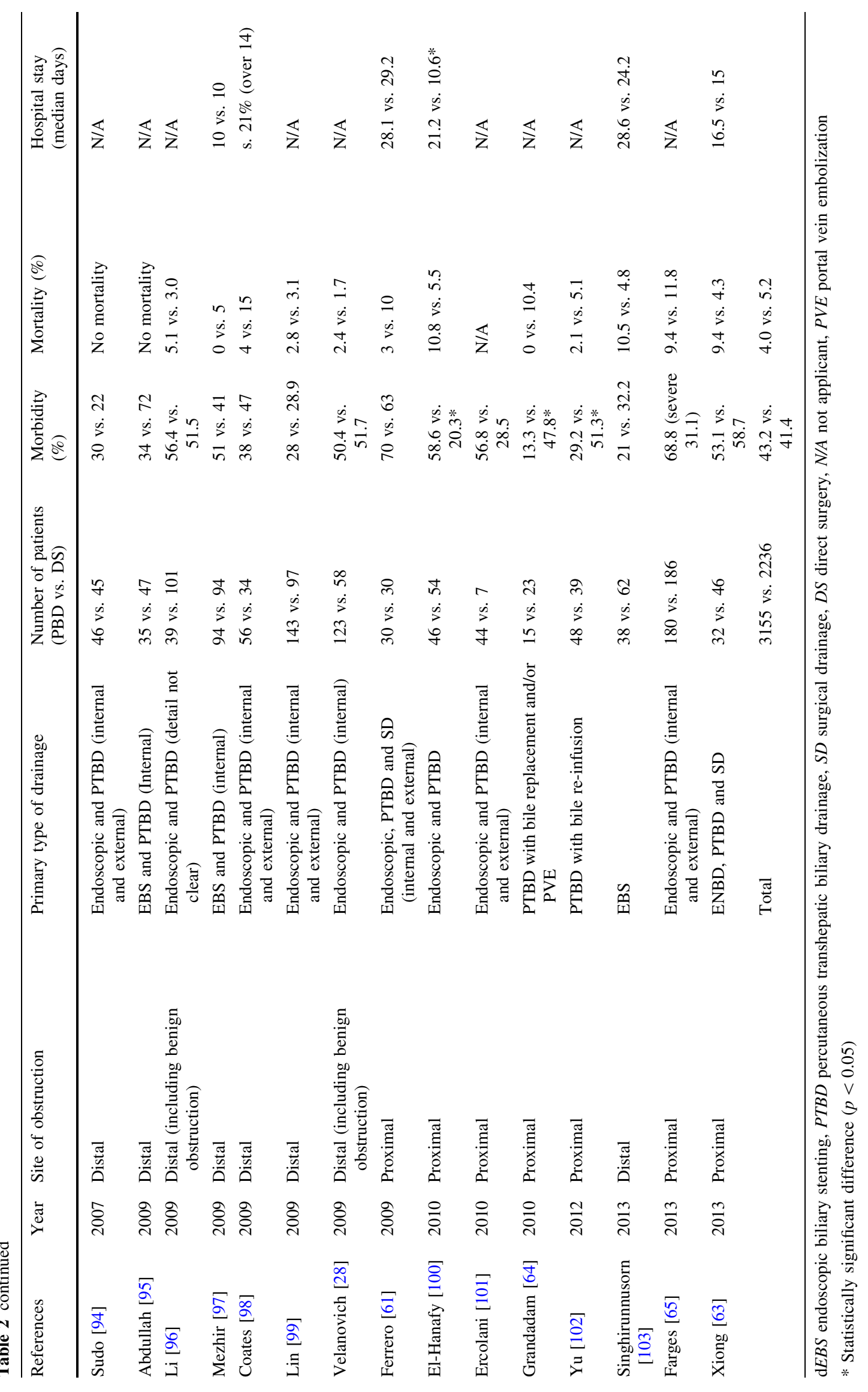




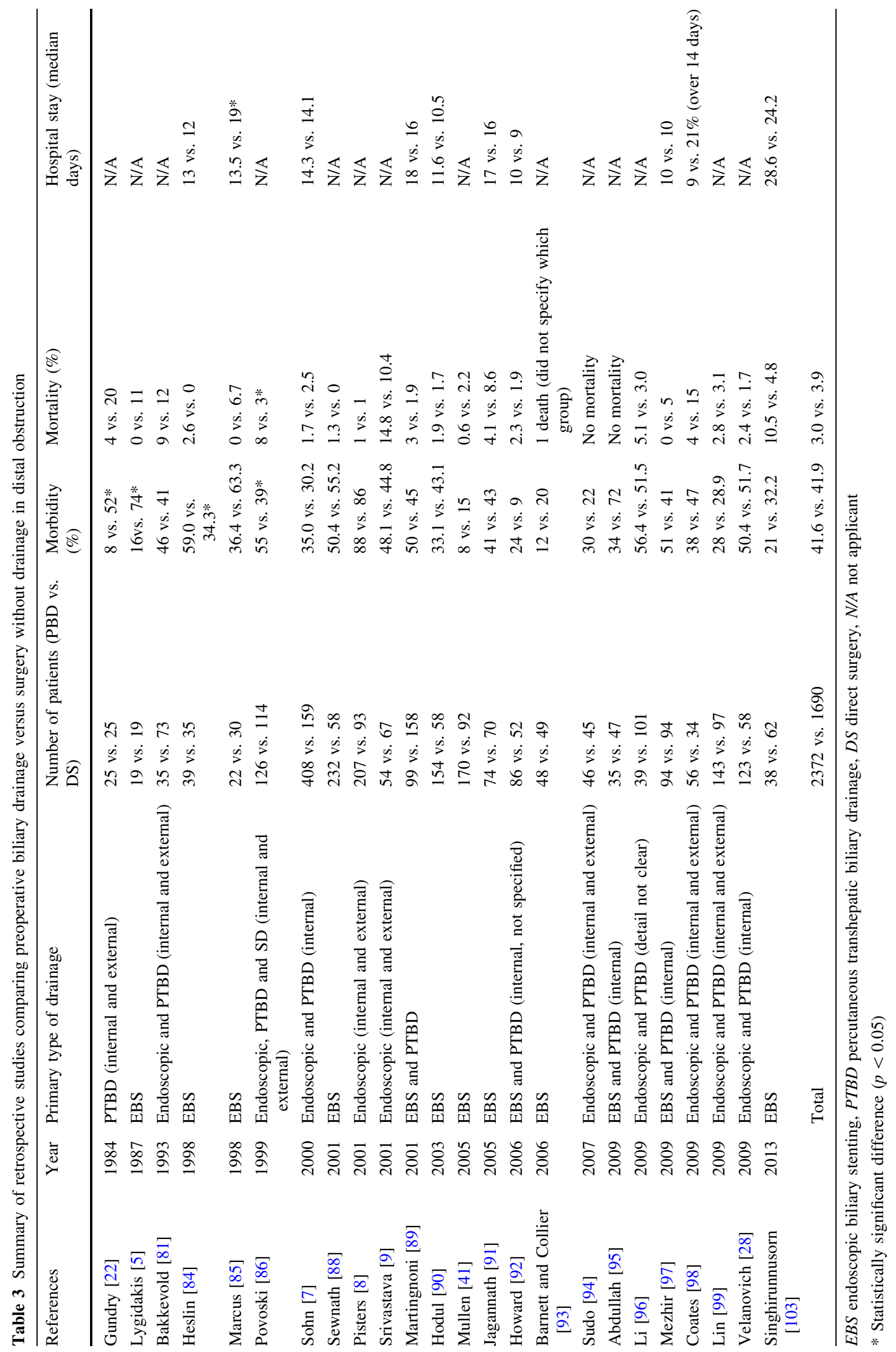


A follow-up study evaluated the relationship between delay in surgery due to PBD and survival [35]. The mean times from randomization to surgery were 5.1 and 1.2 weeks in the PBD and early surgery groups, respectively. In the PBD group, $58 \%$ of the patients underwent resection, compared with $67 \%$ in the early surgery group. The median survival period after randomization in the PBD group was not significantly different from that in the early surgery group (12.7 vs. 12.2 months). The authors concluded that the delay in surgery associated with PBD did not significantly affect the resection rate and survival outcome.

A meta-analysis by Saheh et al., which examined only PBD for distal biliary obstruction undertaken with endoscopic PS placement, found no positive or negative effect of PBD after surgery [36]. PSs cause occlusion owing to the formation of a bacterial biofilm and plant materials [37], resulting in recurrent jaundice frequently with cholangitis and necessitating repeat procedures with stent replacement. Large-bore stents (10-11.5-Fr) have better long-term patency than smaller diameter stents (7-8.5-Fr). Plastic biliary stents do not maintain patency during the time required for most patients to complete neoadjuvant chemoradiotherapy for pancreatic adenocarcinoma [38]. PSs have been typically employed in patients with deemed potentially resectable cancers, and endoscopic placement of SEMSs has been the mainstay of palliation for malignant distal biliary obstructions, primarily in patients with surgically unresectable cancers. SEMSs appear to be superior to PSs for preoperative biliary decompression because of their longer patency rates than PSs owing to the achievement of a threefold greater diameter after deployment [39]. There is concern that SEMSs will negatively affect subsequent curative intent surgery because there may be an inadequate length of the residual bile duct for creation of the choledochojejunal anastomosis. In 2011, Decker et al. [40] reported a retrospective study of 29 patients who underwent PBD for pancreatic cancer (18 PSs, 11 SEMSs). Seven patients (39\%) with PSs required endoscopic reintervention before surgery compared with none of the patients who received a metal stent $(p=0.02)$. Pancreaticoduodenectomy was undertaken successfully in all 29 patients and the presence of a SEMS did not interfere with the successful creation of a biliary anastomosis. Recent observational studies have reported that the use of SEMSs did not increase surgery-associated morbidity or mortality rates [41, 42] and these studies supported their use as a treatment option for delayed surgery or neoadjuvant therapy.

In 2013, Cavell et al. [43] reported the analysis of 593 patients who underwent attempted PD with SEMSs, plastic endoscopic stents, and no stents (NSs). Of these, 509 patients underwent successful PD, of which 71 had SEMSs, 149 had PSs, and 289 had NSs. SEMSs did not increase the overall or serious postoperative complication rate, 30-day mortality rate, length of hospital stay, biliary anastomotic leak, or positive margin. However, SEMSs were associated with more wound infections and longer operative times. The author concluded that the placement of SEMSs is not contraindicated in patients with resectable pancreatic cancer who require PD. In the same year, Siddiqui et al. [44] reported the outcomes of 241 patients with resectable and borderline resectable carcinoma of the pancreatic head treated using SEMSs for PBD. A total of 174/241 (72 \%) patients were deemed to have resectable cancer at the time of diagnosis and underwent surgery. A total of 67 patients with borderline resectable disease underwent neoadjuvant therapy and only $33 \%$ underwent surgery. In all patients, a

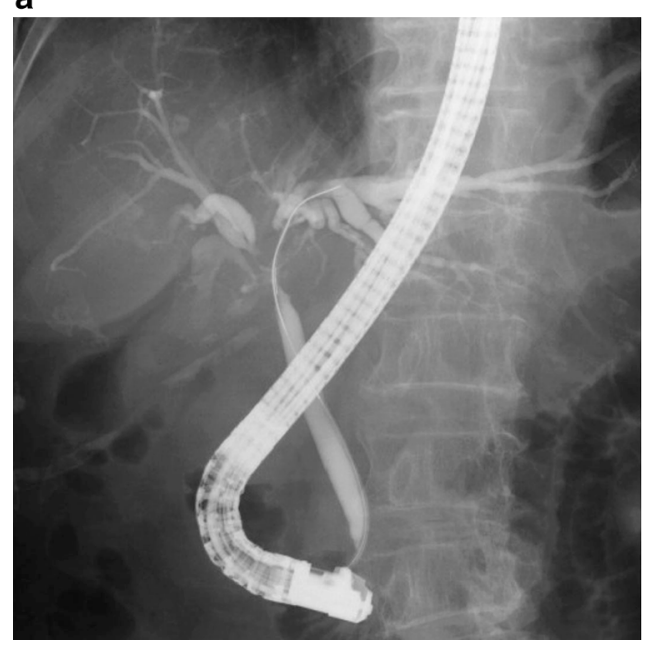

b

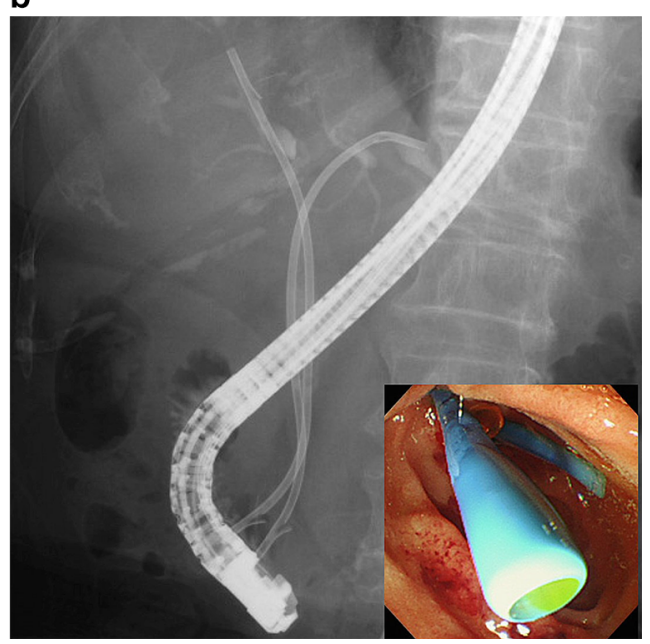

Fig. 3 Cholangiogram shows hilar biliary stricture due to cholangiocarcinoma (a). A fluoroscopic imaging (b) of the two plastic stents placed at the ventral branch of the left lateral segment and the right anterior branch (right lower corner; endoscopic imaging) 
Fig. 4 Cholangiogram shows hilar biliary stricture due to cholangiocarcinoma (a). A fluoroscopic imaging (b) of simultaneous deployment of side-by-side self-expandable metal stents in hilar biliary stricture (right lower corner; endoscopic imaging) a

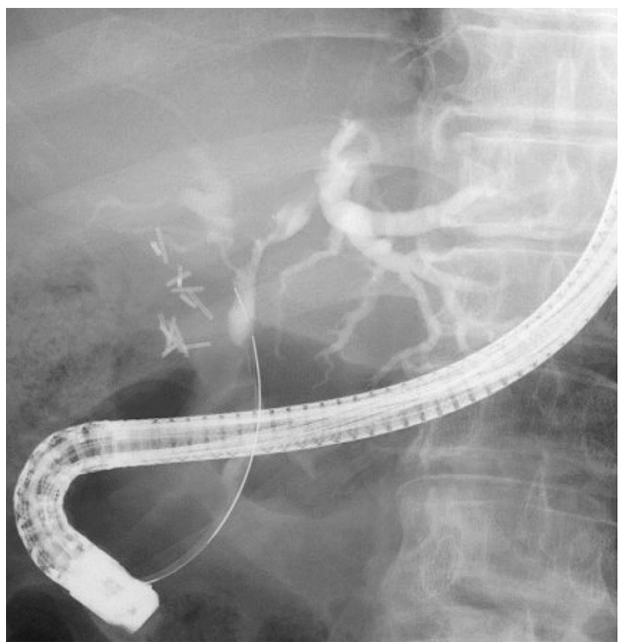

b

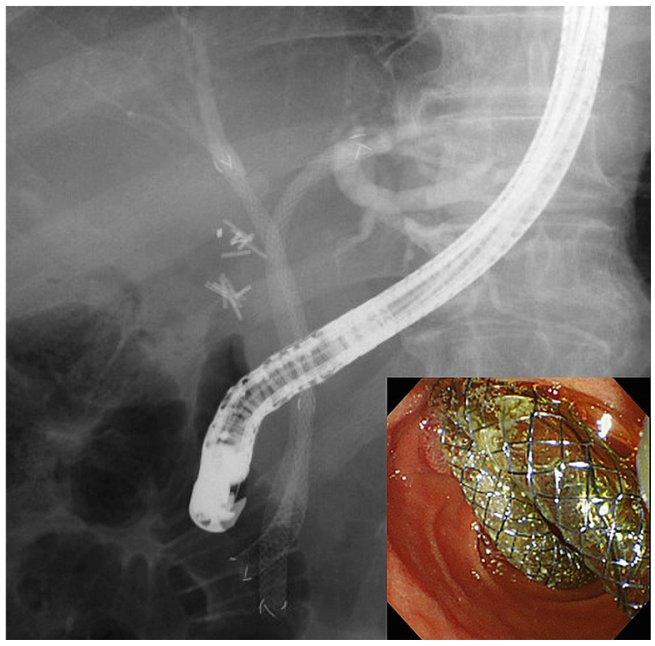

Fig. 5 Schema of classification of hilar cholangiocarcinoma according to Bismuth-Corlette, Type I, II, IIIa, IIIb and IV. Type I tumors are limited to the common hepatic duct, distal to hepatic duct confluence. Type II tumors extend to and involve the hepatic duct confluence. Type IIIa tumors involve the hepatic duct confluence and proximal right hepatic duct. Type IIIb tumors involve the hepatic duct confluence and proximal left hepatic duct. Type IV tumors extend to the bifurcation of both right and left hepatic ducts or multifocal involvemen

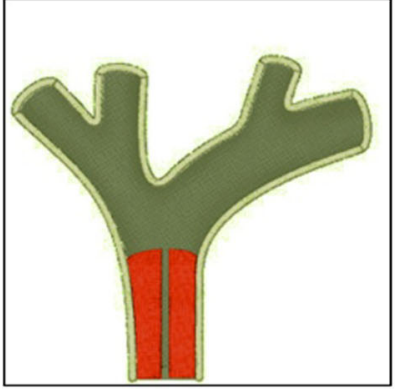

Type I

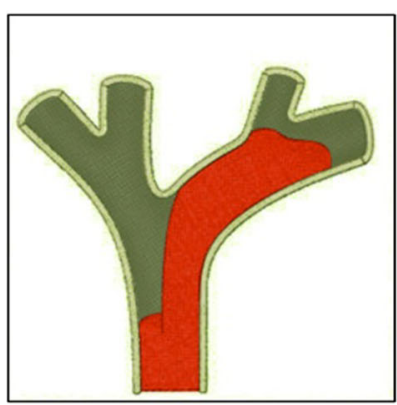

Type IIIB

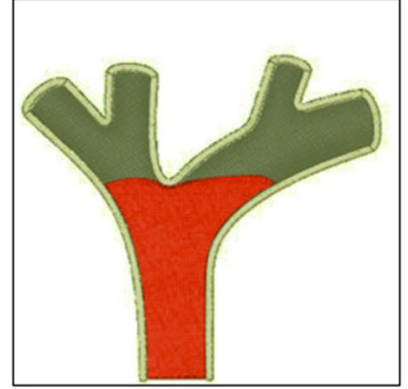

Type II

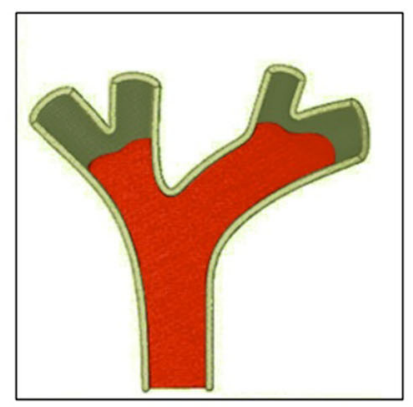

Type IV

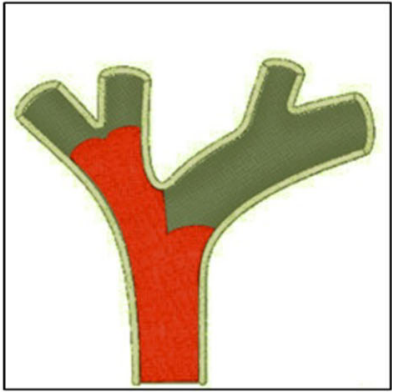

Type IIIA patency, metal stents are more favorable than PSs. To clarify the best preoperative management practice, prospective comparative studies of PBD with metal stents and direct surgery are needed.

\section{PBD for hilar biliary obstruction (proximal obstruction) (Figs. 3a, b, 4a, b)}

Hilar cholangiocarcinoma (HCCA), also known as Klatskin tumor, is a relatively rare tumor with a poor prognosis. Patients with HCCA often suffer from proximal biliary obstruction. The Bismuth-Corlette classification system is 


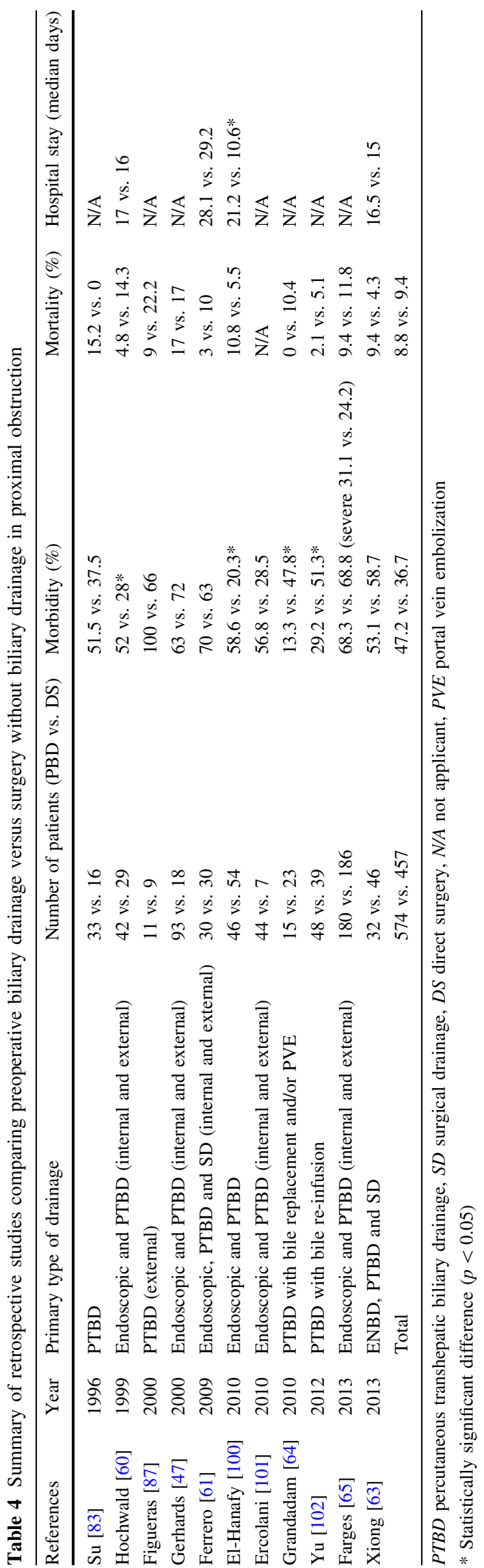

commonly used to provide information regarding tumor location and extent within the biliary tree (Fig. 5) [45]. Radical resection is considered as an optimal treatment; however, it is difficult to obtain negative histological margins because the tumor often infiltrates the portal vein, hepatic artery, and liver parenchyma [46, 47]. A right or left hepatectomy extended to the caudate lobe is mandatory to achieve complete resection with the negative margin. Recently, it has been shown that the resectability rate of HCCA was dependent on the hepatectomy rate [48, 49]. In most studies, long-term results are still deplorable even after radical excision, with the 5-year survival rate ranging from 10 to $40 \%$ [50-52]. Moreover, in patients with HCCA, the postoperative mortality rate is still high at nearly $10 \%$, and the cause of death is mainly hepatic failure [53]. Most patients with HCCA experience cholestasis-induced liver dysfunction, which is implicated as a risk factor of postoperative mortality and morbidity in major hepatic resection [54-57]. Preoperative liver optimization includes portal vein embolization (PVE) and/or PBD, which is thought to be necessary for the safe radical resection of the tumor, mainly extended hemihepatectomy for cholestatic patients [58], despite the absence of a consensus on the appropriate biliary drainage technique and drainage duration. PBD for HCCA has been performed not only for recovering from cholestasis-associated hepatic and systemic toxicity and impaired hepatic regeneration, but also for assessing more precise information about the extent of cancer along the bile duct and the local anatomy of the hepatic hilum involved. In addition, recent advances in imaging technology largely contribute to the evaluation of the relationship between the tumor and the hepatic vessels, and the estimation of the future liver remnant (FLR) volume and liver function.

There are few RCTs or meta-analyses evaluating systematically the clinical benefits of PBD in the surgical resection of HCCA (Table 1). The cited Cochrane review [26] showed no significant difference in the perioperative mortality rate but reported an increased serious morbidity rate in the PBD group; however, the number of patients affected by perihilar cholangiocarcinoma was small and fewer patients underwent liver resection. A recent Chinese systematic review [59] consisting of 11 retrospective studies published between 1996 and 2010 showed no evidence of any clinical benefit of PBD in jaundiced patients with HCCA who were scheduled for surgery. The authors of this previous review investigated the effects of PBD on postoperative morbidity, in-hospital mortality, infectious complication rate, and postoperative hospital stay. In their postoperative complication analysis, comparing 442 patients who underwent PBD with 233 patients who did not undergo drainage, the odds ratio for postoperative morbidity was 1.67 (95\% CI 1.17-2.39). In the postoperative 
mortality analysis, comparing 134 patients who underwent PBD with 122 patients without PBD, the odds ratio for infectious morbidity was 2.17 (95 \% CI 1.24-3.80). In the postoperative hospital stay analysis estimation, 84 patients who underwent PBD were compared with 65 patients who did not undergo PBD; the weighted mean difference was 5.37 days (95\% CI -1.78 to 12.52 days). There was no significant difference in the postoperative mortality rate and hospital stay between the two groups, whereas the overall postoperative complication rate and postoperative infectious complication rate were markedly adversely affected by PBD.

The utility of PBD has been under debate over the past few decades, with some authors in favor of PBD and some others against (Tables 2, 4). Hochwald et al. [60] reported on 71 patients with HCCA. Among them, 42 patients had stent placement in the preoperative period, whereas 29 had none. Hochwald et al. found that preoperative biliary stenting in HCCA regardless of endoscopic or percutaneous transhepatic placement, increased the incidence of contaminated bile and postoperative infectious complications. However, there was no increased risk for the length of hospital stay or mortality in patients with stents. In a retrospective study of $60 \mathrm{HCCA}$ patients who were candidates for liver resection (PTBD in 30 patients), Ferrero et al. [61] reported a significantly higher infection-related morbidity rate in the PBD group (53 vs. $17 \%, p=0.02$ ) including wound infection intra-abdominal abscess, cholangitis, sepsis, and lung infections. Cherqui et al. [62] reported a case-comparison study on major liver resection for HCCA consisting of 20 patients with and 27 patients without jaundice. The perioperative outcome of these patients was compared with that of patients without biliary obstruction. Although no significant differences were noted in the mortality rate ( 5 vs. $0 \%$ ), liver failure rate (5 vs. $0 \%$ ), or postoperative hepatic synthetic function, a significantly higher morbidity rate was encountered in jaundiced patients (50 vs. $15 \%, p=0.006$ ). The complications reported in the jaundiced patients were subphrenic abscess ( $p=0.02)$ and biliary fistula $(p=0.04)$. On the basis of these results, they concluded that major liver resections without PBD were safe in most patients with obstructive jaundice. In a retrospective study comparing 78 jaundiced patients with HCCA who underwent major hepatectomy (PBD 32 patients, non-PBD 46 patients), Xiong et al. observed that the overall postoperative morbidity and mortality rates were not improved by PBD [63], whereas a preoperative serum bilirubin level greater than $10 \mathrm{mg} / \mathrm{dl}$ was found to be one of the independent risk factors for postoperative complications.

In 2010, Garadadam et al. [64] reported a study that compared two groups of patients with Bismuth type III HCCA; 23 patients were operated on without preoperative liver optimization and 15 with preoperative liver optimization (3 PVE, 2 PTBD, and 10 PVE + PTBD). They concluded that preoperative optimization in HCCA Type III reduced the incidence of intra-abdominal abscesses; however, its impact on postoperative survival remains unclear.

In 2013, Farges et al. [65] published the results of a multicenter study of PBD for HCCA involving 366 patients who underwent formal or extended right or left hepatectomy. They evaluated the 90-day mortality rate, morbidity rate, and cause of death, and found that PBD had no effect on the overall postoperative mortality rate; however, PBD was significantly associated with a decreased mortality rate after right hepatectomy (adjusted OR 0.29, 0.11-0.77; $p=0.013$ ) and an increased mortality rate after left hepatectomy (adjusted OR 4.06, 1.01-16.30; $p=0.035$ ). Also, a preoperative serum bilirubin level greater than $2.9 \mathrm{mg} / \mathrm{dl}$ was associated with a significantly increased mortality rate, but only after right hepatectomy (adjusted OR 7.02, 1.73-28.52; $p=0.002$ ). The author concluded that PBD had no effect on the overall mortality of jaundiced patients with HCCA; however, there might be a difference between patients undergoing right-sided and left-sided hepatectomy. Gouma [66] issued a brief comment on this paper advocating the authors' suggestion of the use of PBD in jaundiced patients before right-sided hepatectomy, but not before left-sided resection. The reason for this is that PBD of the left liver is technically easier than drainage of the right lobe owing to the more straightforward anatomy of the left biliary tree. Right-lobe PBD is more often associated with persistent segmental obstruction and infection, and with procedural complications. Furthermore, a substantial liver remnant after left hepatectomy may explain the good results for left hepatectomy without PBD.

Several reports in the Japanese literature have described that PBD particularly with single endoscopic nasobiliary drainage (ENBD) in a future remnant lobe was a favorable preoperative management technique [67-70]. Kawakami et al. [69] reported the outcome of 128 consecutive patients who underwent $\mathrm{PBD}$ before the surgical resection of HCCA. In the enrolled patients, ENBD was performed in 60 patients (57 with unilateral drainage), EBS in 20 patients (15 with unilateral), and PTBD in 48 patients (35 with unilateral). Complications were significantly more frequent in the EBS group than in either the ENBD or PTBD group $(p<0.05)$. Patients in the PTBD group experienced serious complications including vascular injury (8\%) and cancer dissemination (4\%), whereas patients in the endoscopic drainage group had mild postERCP pancreatitis (5\%). There was no significant difference in postsurgical morbidity or mortality among the three groups. These results support ENBD as a preferred PBD method for HCCA. However, some radiologists argued 
against these reported methods. Wiggers et al. [71] espoused the non-inferiority of PTBD over ENBD in terms of the success rate and rare seeding rate. Also, Hwang et al. [72] reported a study from Korea which included 231 patients undergoing PTBD and found a much lower incidence of implantation metastases (i.e., $1.7 \%$ ). They stated that there is no definitive reason for avoiding PTBD when indicated. Furthermore, patients undergoing ENBD may experience severe discomfort with the nasocatheter placement and prolonged hospital stay.

Another issue concerning PBD for HCCA is whether drainage should be unilateral or bilateral. Ishizawa et al. [73] conducted a retrospective cohort study and investigated 42 consecutive patients who underwent selective biliary drainage (SBD) or total biliary drainage (TBD) before hepatectomy. In association with PVE, SBD was superior to TBD in promoting hypertrophy of the FLR, whereby extended hemihepatectomy could be performed more safely. Although not in the perspective of PBD, in an RCT comparing unilateral versus bilateral drainage for patients with unresectable perihilar tumors, De Palma et al. [74] found that unilateral drainage had a higher technical success rate of stent insertion and a significantly lower incidence of complications, mainly early cholangitis. According to these studies, unilateral drainage for the FLR may be adequate for relieving jaundice in most cases, and drainage of the liver to be resected should be limited to cases with uncontrollable cholangitis of the undrained area.

As a new paradigm, Grünhagen et al. [75] reported on the outcomes of the bridge to surgery using metal stents in HCCA. They evaluated 35 patients who presented with operable HCCA. Of these 35 patients, 27 had obstructive jaundice. Ten patients were initially treated using SEMSs and 17 patients initially received PSs. Of the patients who received PSs, 7 had stent occlusion prior to surgery, whereas the patients treated with SEMSs did not experience stent failure. The authors concluded that SEMSs provide adequate biliary drainage and do not preclude subsequent curative surgery in selected patients.

In conclusion, the majority of patients with proximal biliary obstruction who are candidates for major hepatic or biliohepatic resection require $\mathrm{PBD}$, although the drainage route, site, and devices must be tailored to the planned surgical procedures. Selective biliary drainage for the FLR should be applied before PVE. PBD must be limited to the FLR, and the drainage of contralateral liver segments should be applied in cases of segmental cholangitis. ENBD might be the most favorable PBD method with a lower incidence of cholangitis and cancer dissemination along the percutaneous tract, tolerating the drawbacks of inconvenience and discomfort of nasocatheter placement and prolonged preoperative hospitalization. EBS may be useful in establishing internal biliary drainage, although the rate of infectious complication is high. EBS and/or ENBD should be converted to selective PTBD whenever necessary. PBD with metal stents for bridging to surgery is possibly promising in selected cases. Currently, the preference for PBD largely depends on local expertise. Prospective randomized studies with a large sample size are needed to establish the optimal mode of PBD and to evaluate the potential benefits of PBD in patients with HCCA.

In summary, there is as yet no optimal PBD method regardless of the distal and proximal biliary strictures because of the small sample size and the lack of better control groups in previous studies. Thus, prospective randomized studies with a large sample size are needed to establish the optimal mode of PBD and to evaluate the potential benefits of PBD in patients with both distal biliary obstruction and HCCA.

Conflict of interest The authors declare that they have no conflict of interest.

\section{References}

1. van der Gaag NA, Kloek JJ, de Castro SM, et al. Preoperative biliary drainage in patients with obstructive jaundice: history and current status. J Gastrointest Surg. 2009;13:814-20.

2. Klinkenbijl JH, Jeekel J, Schmitz PI, et al. Carcinoma of the pancreas and periampullary region: palliation versus cure. $\mathrm{Br} \mathbf{J}$ Surg. 1993;80:1575-8.

3. Trede M, Schwall G. The complications of pancreatectomy. Ann Surg. 1988;207:39-47.

4. Kimmings AN, van Deventer SJ, Obertop H, et al. Endotoxin, cytokines, and endotoxin binding proteins in obstructive jaundice and after preoperative biliary drainage. Gut. 2000;46:725-31.

5. Lygidakis NJ, van der Heyde MN, Lubbers MJ, et al. Evaluation of preoperative biliary drainage in the surgical management of pancreatic head carcinoma. Acta Chir Scand. 1987;153:665-8.

6. Povoski SP, Karpeh MS Jr, Conlon KC, et al. Association of preoperative biliary drainage with postoperative outcome following pancreaticoduodenectomy. Ann Surg. 1999;230:131-42.

7. Sohn TA, Yeo CJ, Cameron JL et al. Do preoperative biliary stents increase postpancreaticoduodenectomy complications? J Gastrointest Surg. 2000; 4:258-67 (discussion 267-8).

8. Pisters PW, Hudec WA, Hess KR, et al. Effect of preoperative biliary decompression on pancreaticoduodenectomy-associated morbidity in 300 consecutive patients. Ann Surg. 2001;234:47-55.

9. Srivastava S, Sikora SS, Kumar A, et al. Outcome following pancreaticoduodenectomy in patients undergoing preoperative biliary drainage. Dig Surg. 2001;18:381-7.

10. Jinkins LJ, Parmar AD, Han Y, et al. Current trends in preoperative biliary stenting in patients with pancreatic cancer. Surgery. 2013;154:179-89.

11. Speer AG, Cotton PB, Russell RC, et al. Randomised trial of endoscopic versus percutaneous stent insertion in malignant obstructive jaundice. Lancet. 1987;2:57-62.

12. Herzog T, Belyaev O, Muller CA, et al. Bacteribilia after preoperative bile duct stenting: a prospective study. J Clin Gastroenterol. 2009;43:457-62. 
13. Engelken FJ, Bettschart V, Rahman MQ, et al. Prognostic factors in the palliation of pancreatic cancer. Eur J Surg Oncol. 2003;29:368-73.

14. Michelassi F, Erroi F, Dawson PJ, et al. Experience with 647 consecutive tumors of the duodenum, ampulla, head of the pancreas, and distal common bile duct. Ann Surg. 1989;210:544-54 (discussion 554-6).

15. Smith RA, Bosonnet L, Ghaneh P, et al. The platelet-lymphocyte ratio improves the predictive value of serum CA19-9 levels in determining patient selection for staging laparoscopy in suspected periampullary cancer. Surgery. 2008;143:658-66.

16. Doucas H, Sutton CD, Zimmerman A, et al. Assessment of pancreatic malignancy with laparoscopy and intraoperative ultrasound. Surg Endosc. 2007;21:1147-52.

17. Garcea G, Dennison AR, Pattenden CJ, et al. Survival following curative resection for pancreatic ductal adenocarcinoma. A systematic review of the literature. JOP. 2008;9:99-132.

18. Greve JW, Gouma DJ, Soeters PB, et al. Suppression of cellular immunity in obstructive jaundice is caused by endotoxins: a study with germ-free rats. Gastroenterology. 1990;98:478-85.

19. Gouma DJ, Coelho JC, Fisher JD, et al. Endotoxemia after relief of biliary obstruction by internal and external drainage in rats. Am J Surg. 1986;151:476-9.

20. Denning DA, Ellison EC, Carey LC. Preoperative percutaneous transhepatic biliary decompression lowers operative morbidity in patients with obstructive jaundice. Am J Surg. 1981;141:61-5.

21. Norlander A, Kalin B, Sundblad R. Effect of percutaneous transhepatic drainage upon liver function and postoperative mortality. Surg Gynecol Obstet. 1982;155:161-6.

22. Gundry SR, Strodel WE, Knol JA, et al. Efficacy of preoperative biliary tract decompression in patients with obstructive jaundice. Arch Surg. 1984;119:703-8.

23. Neff RA, Fankuchen EI, Cooperman AM, et al. The radiological management of malignant biliary obstruction. Clin Radiol. 1983;34:143-6.

24. Smith RC, Pooley M, George CR, et al. Preoperative percutaneous transhepatic internal drainage in obstructive jaundice: a randomized, controlled trial examining renal function. Surgery. 1985;97:641-8.

25. Strasberg SM, Gao F, Sanford D, et al. Jaundice: an important, poorly recognized risk factor for diminished survival in patients with adenocarcinoma of the head of the pancreas. HPB (Oxford). 2014;16:150-6.

26. Fang Y, Gurusamy KS, Wang Q, et al. Pre-operative biliary drainage for obstructive jaundice. Cochrane Database Syst Rev. 2012;9:CD005444.

27. Garcea G, Chee W, Ong SL, et al. Preoperative biliary drainage for distal obstruction: the case against revisited. Pancreas. 2010;39:119-26.

28. Velanovich V, Kheibek T, Khan M. Relationship of postoperative complications from preoperative biliary stents after pancreaticoduodenectomy. A new cohort analysis and meta-analysis of modern studies. JOP. 2009;10:24-9.

29. van der Gaag NA, Rauws EA, van Eijck $\mathrm{CH}$, et al. Preoperative biliary drainage for cancer of the head of the pancreas. N Engl J Med. 2010;362:129-37.

30. Chen VK, Arguedas MR, Baron TH. Expandable metal biliary stents before pancreaticoduodenectomy for pancreatic cancer: a Monte-Carlo decision analysis. Clin Gastroenterol Hepatol. 2005;3:1229-37.

31. Soderlund C, Linder S. Covered metal versus plastic stents for malignant common bile duct stenosis: a prospective, randomized, controlled trial. Gastrointest Endosc. 2006;63:986-95.

32. Kaassis M, Boyer J, Dumas R, et al. Plastic or metal stents for malignant stricture of the common bile duct? Results of a randomized prospective study. Gastrointest Endosc. 2003;57:178-82.

33. Isayama H, Nakai Y, Kogure H, et al. Biliary self-expandable metallic stent for unresectable malignant distal biliary obstruction: which is better: covered or uncovered? Dig Endosc. 2013;25(Suppl 2):71-4.

34. Baron TH, Kozarek RA. Preoperative biliary stents in pancreatic cancer-proceed with caution. N Engl J Med. 2010;362:170-2.

35. Eshuis EJ, van der Gaag NA, Rauws EA, et al. Therapeutic delay and survival after surgery for cancer of the pancreatic head with or without preoperative biliary drainage. Ann Surg. 2010;252:840-9.

36. Saleh MM, Nørregaard P, Jørgensen HL, et al. Preoperative endoscopic stent placement before pancreaticoduodenectomy: a meta-analysis of the effect on morbidity and mortality. Gastrointest Endosc. 2002;56:529-34.

37. van Berkel AM, van Marle J, Groen AK, et al. Mechanisms of biliary stent clogging: confocal laser scanning and scanning electron microscopy. Endoscopy. 2005;37:729-34.

38. Boulay BR, Gardner TB, Gordon SR. Occlusion rate and complications of plastic biliary stent placement in patients undergoing neoadjuvant chemoradiotherapy for pancreatic cancer with malignant biliary obstruction. J Clin Gastroenterol. 2010;44:452-5.

39. Moss AC, Morris E, Leyden J, et al. Malignant distal biliary obstruction: a systematic review and meta-analysis of endoscopic and surgical bypass results. Cancer Treat Rev. 2007;33:213-21.

40. Decker C, Christein JD, Phadnis MA, et al. Biliary metal stents are superior to plastic stents for preoperative biliary decompression in pancreatic cancer. Surg Endosc. 2011;25:2364-7.

41. Mullen JT, Lee JH, Gomez HF, et al. Pancreaticoduodenectomy after placement of endobiliary metal stents. Hepatol J Gastrointest Surg. 2005;9:1094-104 (discussion 1104-5).

42. Pop GH, Richter JA, Sauer B, et al. Bridge to surgery using partially covered self-expandable metal stents (PCMS) in malignant biliary stricture: an acceptable paradigm? Surg Endosc. 2011;25:613-8.

43. Cavell LK, Allen PJ, Vinoya C, et al. Biliary self-expandable metal stents do not adversely affect pancreaticoduodenectomy. Am J Gastroenterol. 2013;108:1168-73.

44. Siddiqui AA, Mehendiratta V, Loren D, et al. Self-expanding metal stents (SEMS) for preoperative biliary decompression in patients with resectable and borderline-resectable pancreatic cancer: outcomes in 241 patients. Dig Dis Sci. 2013;58:1744-50.

45. Bismuth H, Corlette MB. Intrahepatic cholangioenteric anastomosis in carcinoma of the hilus of the liver. Surg Gynecol Obstet. 1975;140:170-6.

46. Belghiti J, Ogata S. Preoperative optimization of the liver for resection in patients with hilar cholangiocarcinoma. HPB (Oxford). 2005;7:252-3.

47. Gerhards MF, van Gulik TM, de Wit LT, et al. Evaluation of morbidity and mortality after resection for hilar cholangiocarcinoma-a single center experience. Surgery. 2000;127:395-404.

48. Launois B, Terblanche J, Lakehal M, et al. Proximal bile duct cancer: high resectability rate and 5-year survival. Ann Surg. 1999;230:266-75.

49. Neuhaus P, Jonas S, Settmacher U, et al. Surgical management of proximal bile duct cancer: extended right lobe resection increases resectability and radicality. Langenbecks Arch Surg. 2003;388:194-200

50. Nakeeb A, Pitt HA, Sohn TA, et al. Cholangiocarcinoma. A spectrum of intrahepatic, perihilar, and distal tumors. Ann Surg. 1996;224:463-73 (discussion 473-5). 
51. Cheng JL, Bruno MJ, Bergman JJ, et al. Endoscopic palliation of patients with biliary obstruction caused by nonresectable hilar cholangiocarcinoma: efficacy of self-expandable metallic Wallstents. Gastrointest End's. 2002;56:33-9.

52. Soares KC, Kamel I, Cosgrove DP, et al. Hilar cholangiocarcinoma: diagnosis, treatment options, and management. Hepatobiliary Surg Nutr. 2014;3:18-34.

53. Nagino M, Kamiya J, Uesaka K, et al. Complications of hepatectomy for hilar cholangiocarcinoma. World J Surg. 2001;25:1277-83.

54. Belghiti J, Hiramatsu K, Benoist S, et al. Seven hundred fortyseven hepatectomies in the 1990s: an update to evaluate the actual risk of liver resection. J Am Coll Surg. 2000;191:38-46.

55. Blamey SL, Fearon KC, Gilmour WH, et al. Prediction of risk in biliary surgery. Br J Surg. 1983;70:535-8.

56. Dixon JM, Armstrong CP, Duffy SW, et al. Factors affecting morbidity and mortality after surgery for obstructive jaundice: a review of 373 patients. Gut. 1983;24:845-52.

57. Shigeta H, Nagino M, Kamiya J, et al. Bacteremia after hepatectomy: an analysis of a single-center, 10-year experience with 407 patients. Langenbecks Arch Surg. 2002;387:117-24.

58. Kawasaki S, Imamura H, Kobayashi A, et al. Results of surgical resection for patients with hilar bile duct cancer: application of extended hepatectomy after biliary drainage and hemihepatic portal vein embolization. Ann Surg. 2003;238:84-92.

59. Liu F, Li Y, Wei Y, et al. Preoperative biliary drainage before resection for hilar cholangiocarcinoma: whether or not? A systematic review. Dig Dis Sci. 2011;56:663-72.

60. Hochwald SN, Burke EC, Jarnagin WR, et al. Association of preoperative biliary stenting with increased postoperative infectious complications in proximal cholangiocarcinoma. Arch Surg. 1999; 134:261-6.

61. Ferrero A, Lo Tesoriere R, Viganò L, et al. Preoperative biliary drainage increases infectious complications after hepatectomy for proximal bile duct tumor obstruction. World J Surg. 2009;33:318-25.

62. Cherqui D, Benoist S, Malassagne B, et al. Major liver resection for carcinoma in jaundiced patients without preoperative biliary drainage. Arch Surg. 2000;135:302-8.

63. Xiong JJ, Nunes QM, Huang W, et al. Preoperative biliary drainage in patients with hilar cholangiocarcinoma undergoing major hepatectomy. World J Gastroenterol. 2013;19:8731-9.

64. Grandadam S, Compagnon P, Arnaud A, et al. Role of preoperative optimization of the liver for resection in patients with hilar cholangiocarcinoma type III. Ann Surg Oncol. 2010;17:3155-61.

65. Farges O, Regimbeau JM, Fuks D, et al. Multicentre European study of preoperative biliary drainage for hilar cholangiocarcinoma. Br J Surg. 2013;100:274-83.

66. Gouma DJ. Multicentre European study of preoperative biliary drainage for hilar cholangiocarcinoma (Br J Surg 2013; 100: 274-283). Br J Surg. 2013;100:283-4.

67. Maguchi H, Takahashi K, Katanuma A, et al. Preoperative biliary drainage for hilar cholangiocarcinoma. J Hepatobiliary Pancreat Surg. 2007;14:441-6.

68. Arakura N, Takayama M, Ozaki Y, et al. Efficacy of preoperative endoscopic nasobiliary drainage for hilar cholangiocarcinoma. J Hepatobiliary Pancreat Surg. 2009;16:473-7.

69. Kawakami H, Kuwatani M, Onodera M, et al. Endoscopic nasobiliary drainage is the most suitable preoperative biliary drainage method in the management of patients with hilar cholangiocarcinoma. J Gastroenterol. 2011;46:242-8.

70. Kawashima H, Itoh A, Ohno E, et al. Preoperative endoscopic nasobiliary drainage in 164 consecutive patients with suspected perihilar cholangiocarcinoma: a retrospective study of efficacy and risk factors related to complications. Ann Surg. 2013;257:121-7.

71. Wiggers JK, Rauws EA, Gouma DJ, et al. Preoperative endoscopic nasobiliary drainage in patients with suspected hilar cholangiocarcinoma; better than endoscopic or percutaneous biliary drainage? Ann Surg. 2014. doi:10.1097/SLA. 0000000000000521.

72. Hwang S, Song GW, Ha TY. Reappraisal of percutaneous transhepatic biliary drainage tract recurrence after resection of perihilar bile duct cancer. World J Surg. 2012;36:379-85.

73. Ishizawa $\mathrm{T}$, Hasegawa $\mathrm{K}$, Sano $\mathrm{K}$, et al. Selective versus total biliary drainage for obstructive jaundice caused by a hepatobiliary malignancy. Am J Surg. 2007;193:149-54.

74. De Palma GD, Galloro G, Siciliano S, et al. Unilateral versus bilateral endoscopic hepatic duct drainage in patients with malignant hilar biliary obstruction: results of a prospective, randomized, and controlled study. Gastrointest Endosc. 2001;53:547-53.

75. Grünhagen DJ, Dunne DF, Sturgess RP, et al. Metal stents: a bridge to surgery in hilar cholangiocarcinoma. HPB (Oxford). 2013;15:372-8.

76. Hatfield AR, Tobias R, Terblanche J, et al. Preoperative external biliary drainage in obstructive jaundice. A prospective controlled clinical trial. Lancet. 1982;2:896-9.

77. McPherson GA, Benjamin IS, Hodgson HJ, et al. Pre-operative percutaneous transhepatic biliary drainage: the results of a controlled trial. Br J Surg. 1984;71:371-5.

78. Pitt HA, Gomes AS, Lois JF, et al. Does preoperative percutaneous biliary drainage reduce operative risk or increase hospital cost? Ann Surg. 1985;201:545-53.

79. Lai EC, Mok FP, Fan ST, et al. Preoperative endoscopic drainage for malignant obstructive jaundice. Br J Surg. 1994;81:1195-8.

80. Wig JD, Kumar H, Suri S, et al. Usefulness of percutaneous transhepatic biliary drainage in patients with surgical jaundicea prospective randomised study. $\mathrm{J}$ Assoc Physicians India. 1999;47:271-4.

81. Bakkevold KE, Kambestad B. Morbidity and mortality after radical and palliative pancreatic cancer surgery. Risk factors influencing the short-term results. Ann Surg. 1993;217:356-68.

82. Karsten TM, Allema JH, Reinders M, et al. Preoperative biliary drainage, colonisation of bile and postoperative complications in patients with tumours of the pancreatic head: a retrospective analysis of 241 consecutive patients. Eur J Surg. 1996;162:881-8.

83. Su CH, Tsay SH, Wu CC, et al. Factors influencing postoperative morbidity, mortality, and survival after resection for hilar cholangiocarcinoma. Ann Surg. 1996;223:384-94.

84. Heslin MJ, Brooks AD, Hochwald SN, et al. A preoperative biliary stent is associated with increased complications after pancreatoduodenectomy. Arch Surg. 1998;133:149-54.

85. Marcus SG, Dobryansky M, Shamamian P, et al. Endoscopic biliary drainage before pancreaticoduodenectomy for periampullary malignancies. J Clin Gastroenterol. 1998;26:125-9.

86. Povoski SP, Karpeh MS Jr, Conlon KC, et al. Preoperative biliary drainage: impact on intraoperative bile cultures and infectious morbidity and mortality after pancreaticoduodenectomy. J Gastrointest Surg. 1999;3:496-505.

87. Figueras J, Llado L, Valls C, et al. Changing strategies in diagnosis and management of hilar cholangiocarcinoma. Liver Transpl. 2000;6:786-94.

88. Sewnath ME, Birjmohun RS, Rauws EA, et al. The effect of preoperative biliary drainage on postoperative complications after pancreaticoduodenectomy. J Am Coll Surg. 2001;192:726-34.

89. Martignoni ME, Wagner M, Krähenbühl L, et al. Effect of preoperative biliary drainage on surgical outcome after pancreatoduodenectomy. Am J Surg. 2001;181:52-9 (discussion 87). 
90. Hodul P, Creech S, Pickleman J, et al. The effect of preoperative biliary stenting on postoperative complications after pancreaticoduodenectomy. Am J Surg. 2003;186:420-5.

91. Jagannath P, Dhir V, Shrikhande S, et al. Effect of preoperative biliary stenting on immediate outcome after pancreaticoduodenectomy. Br J Surg. 2005;92:356-61.

92. Howard TJ, Yu J, Greene RB, et al. Influence of bactibilia after preoperative biliary stenting on postoperative infectious complications. J Gastrointest Surg. 2006;10:523-31.

93. Barnett SA, Collier NA. Pancreaticoduodenectomy: does preoperative biliary drainage, method of pancreatic reconstruction or age influence perioperative outcome? A retrospective study of 104 consecutive cases. ANZ J Surg. 2006;76:563-8.

94. Sudo T, Murakami Y, Uemura K, et al. Specific antibiotic prophylaxis based on bile cultures is required to prevent postoperative infectious complications in pancreatoduodenectomy patients who have undergone preoperative biliary drainage. World J Surg. 2007;31:2230-5.

95. Abdullah SA, Gupta T, Jaafar KA, et al. Ampullary carcinoma: effect of preoperative biliary drainage on surgical outcome. World J Gastroenterol. 2009;15:2908-12.

96. Li Z, Zhang Z, Hu W, et al. Pancreaticoduodenectomy with preoperative obstructive jaundice: drainage or not. Pancreas. 2009;38:379-86.

97. Mezhir JJ, Brennan MF, Baser RE, et al. A matched case-control study of preoperative biliary drainage in patients with pancreatic adenocarcinoma: routine drainage is not justified. J Gastrointest Surg. 2009;13:2163-9.

98. Coates JM, Beal SH, Russo JE, et al. Negligible effect of selective preoperative biliary drainage on perioperative resuscitation, morbidity, and mortality in patients undergoing pancreaticoduodenectomy. Arch Surg. 2009;144:841-7.

99. Lin SC, Shan YS, Lin PW. Adequate preoperative biliary drainage is determinative to decrease postoperative infectious complications after pancreaticoduodenectomy. Hepatogastroenterology. 2010;57:698-705.

100. El-Hanafy E. Pre-operative biliary drainage in hilar cholangiocarcinoma, benefits and risks, single center experience. Hepatogastroenterology. 2010;57:414-9.

101. Ercolani G, Zanello M, Grazi GL, et al. Changes in the surgical approach to hilar cholangiocarcinoma during an 18-year period in a Western single center. $\mathrm{J}$ Hepatobiliary Pancreat Sci. 2010;17:329-37.

102. Yu FX, Ji SQ, Su LF, et al. Effectiveness and safety of preoperative percutaneous transhepatic cholangiodrainage with bile re-infusion in patients with hilar cholangiocarcinoma: a retrospective controlled study. Am J Med Sci. 2013;346:353-7.

103. Singhirunnusorn J, Roger L, Chopin-Laly X, et al. Value of preoperative biliary drainage in a consecutive series of resectable periampullary lesions. From randomized studies to real medical practice. Langenbecks Arch Surg. 2013;398:295-302. 